\title{
Design of Automatic Test System for a Missile Radar Signals Receiver Based on PC104 Computer
}

\author{
Xiaoyu Zhang, Guifang Cai \\ Department of Aviation Ammunition Engineering, The First Aeronautics College of Air Force, \\ Xinyang,464000,China \\ email: zxydyx1024@126.com
}

Keywords: missile, radar, test, PC104 computer

\begin{abstract}
Radar signals receiver is the important signals processor of a missile, it is important for the missile to transform and process the missile's signals. In order to assure the stability of the radar signals receiver, a kind of automatic test system for a missile radar signals receiver based on PC104 is designed. The PC104 built-in computer is the control core, of the system, the dummy instrument technology and $\mathrm{VC}++6.0$ software are used to design program, the real-time test technology and software hardware combination anti-jamming technology are adopted. After tryout, the results illustrate that the system has such advantages as faults orientation fast, test efficiency high, it has nicer expansibility and good military benefits.
\end{abstract}

\section{Introduction}

Radar signals receiver is the important signals process combination of a missile radio control instrument, it is also the core part of guiding loop, it is important for a missile to transform and process signals. The traditional radar signals receiver auto-test instrument has such shortcomings as faults orientation slow, faults easily and faults diagnosis efficiency low and so on. In order to ameliorate such above shortcomings, a kind of automatic test system for a missile radar signals receiver based on PC104 is designed, such system not only can provide technology support for the missile's faults diagnosis, repair and over life, but also can improve the weapon maintenance and support capability[1].

\section{System Design Principle}

Portability principle. In order to meet the environment's requirements of ground and motorial carriers, the system requires small cubage, low weight and portable.

Function expansibility principle. During portability design, the system's functions and expansibility can't be reduced. The PC104 bus is chosen to build the whole system, the hardware module based on PC104 bus has many advantages such as small cubage, powerful function, good expansibility and so on.

Friendly man-computer alternatively principle. It is important for system's software to have man-computer capability. Based on the dummy instrument technology, the page layouts are shown by dummy instruments, the unnecessary wrong actions can be avoided effectively [2][3].

\section{Whole Design Scheme of System}

The main function of radar receiver is to receive, amplify and process the radar signals which are sent to missile by sub aerial guiding radar, the radar signals are transformed to the missile's flight control instructions by preselecting, amplifying, frequency conversing, flitting, and A/D transforming, they are sent to the autopilot and the radio fuse, the missile's flight stance is controlled, and the missile can be controlled to flight along the reelection ballistic trajectory.

The test system subassembly scheme based on PC104 computer is adopted by the radar receiver auto-test system. In the system, the instruments are supported by general test bus standard; they can 
be used as soon as inserted. Such system has many advantages such as expand easily, standardization degree high, it is the main scheme. The system's core is computer, the main software modules are fixed in the computer. The test-self module can be used to set parameters and test itself statue, and the results are shown to the user, when errors are found, user is suggested to progress [4][5][6].

Under the control of the manage module, The test module can be used to control every hardware unit though drive program the stimulant signals are distributed to the tested objects, by the matrix switches, at the same time, the tested response signals are distributed to the test units by driving units. When tested, the test programs are transferred by the computer, the orders are sent to the tested units, the signals distribution orders are sent to the matrix switches. The test stimulated signals and response signals are sent to the matrix switches to be tested through adapters, at last, the signals analysis results are sent back to the computer. The backtracked results of each test unit are dealt with by the computer, and the last statue evaluation results are got, the work flow chart is shown in the Fig.1.

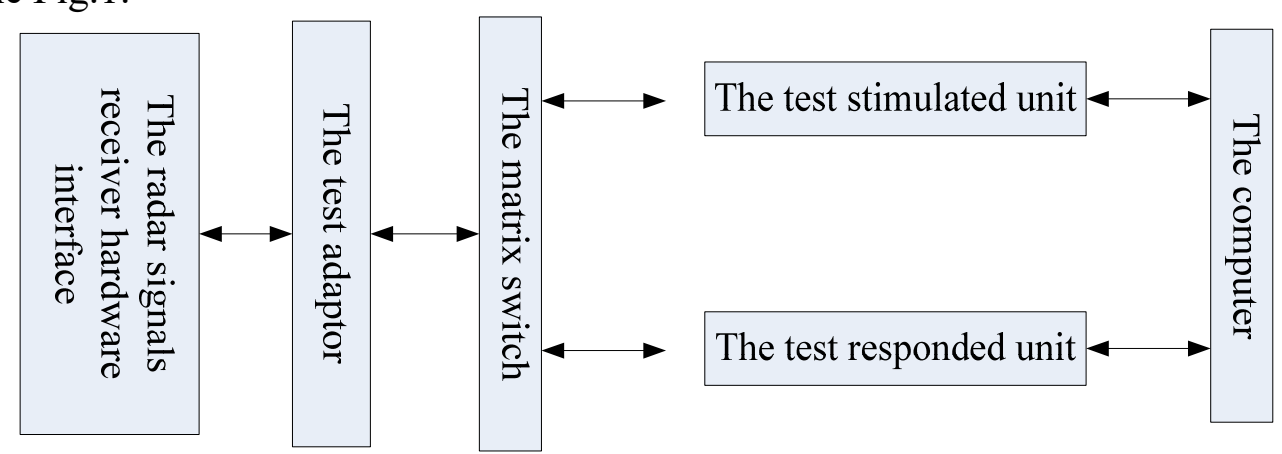

Fig.1. the System Work Floe Chart

\section{System Hardware Design}

The test system is composed of PC104 computer, signals modulator, data acquisition board, LCD screen, power supply module, test cable and so on. The hardware structure is shown in the Fig.2.

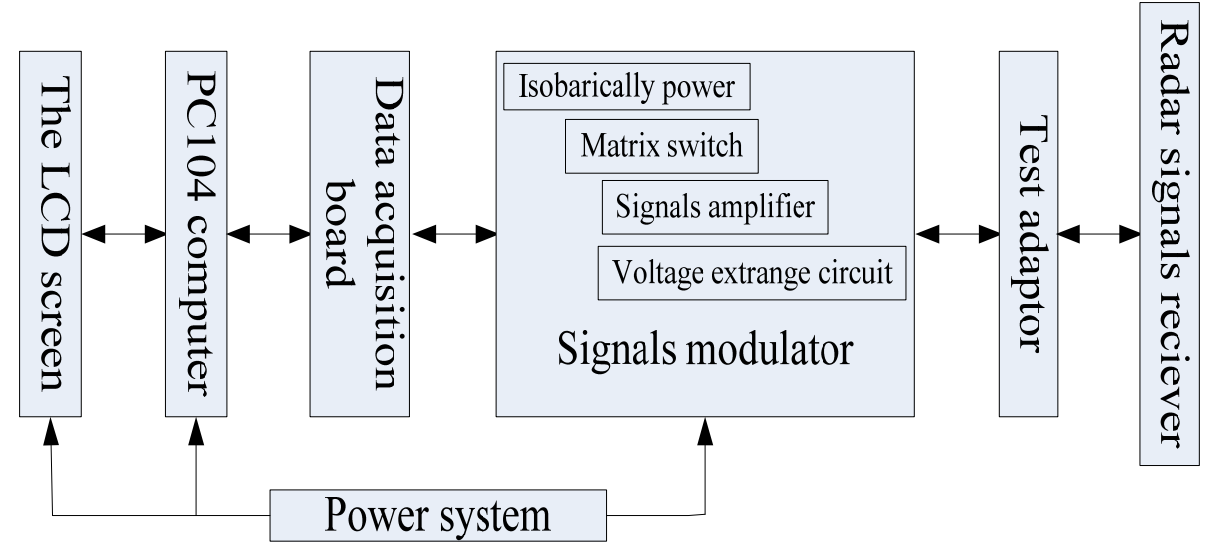

Fig.2. the Test System Hardware Structure

PC104 Computer. The PC104 computer is the whole system's core, it manage all the test tasks, provide good man-computer interface, make test database, maintain ante test data, manage records and print test data. PCM-3586 bus is adopted by pc104 computer, the data exchange between the computer and data acquisition board, and the data can be shown, conserved, faults analysis and so on[7][8].

Data Acquisition Board. ART2010 based on PC104 bus is adapted by data acquisition board, through relevant I/O interface control radar signals receiver electrifying, voltage switching, A/D sampling for tested voltage of signals modulator is completed at the same time. Data acquisition card can be communicated with the computer through PC104 bus [9].

Signals Modulator. The signals modulator has three kinds of functions. The first is to amplify or attenuate the tested voltage signals, and assure that the signals meet the requires of data question 
voltage input range; the second is to receive the $\mathrm{I} / \mathrm{O}$ control instructions which outputted by data acquisition, the analog signals or revenant test parameters are chosen by exchanging matrix switch; the third is to produce the necessary analog signals. AD/DC modules provide needed power for every circuit, and provide the power for test system when tested [10][11].

The signals modulators composed of isobarically power circuit, electrical switch matrix, signals amplifier, voltage extra circuit, coding circuit, power extranging circuit and so on. Its functions are as follow. It can complete revenant tested channels switching according to the test instructions, it can change resistances test to voltage test by using the isobarically power, the modulated test voltage can be completed the A/D sampling through data acquisition board.

Power System. The power system is composed of 24-28DC-CD module, 24-12DC-C module and $24-05 \mathrm{DC}-\mathrm{CD}$ module, the input $\mathrm{AC}$ is $220 \mathrm{~V} / 50 \mathrm{~Hz}$, the output $\mathrm{DC}$ is $+28 \mathrm{~V},+24 \mathrm{~V},+12 \mathrm{~V}$ and $+5 \mathrm{~V}$, it can provide $+28 \mathrm{~V},+24 \mathrm{~V}$ and $+5 \mathrm{~V}$ DC for radar signals receiver, it can provide $+12 \mathrm{~V}$ and $+5 \mathrm{~V}$ DC for PC104 computer, it can provide $+12 \mathrm{~V}$ DC for the screen.

Test Adapter. The test adapter is a bridge between quote-test system and radar signals receiver, it is composed of the adapter machine boxes and signals commutate circuit board.

The functions of adapter is to bring every stimulated signal, to amply, separated, flitting and distributing the tested signals, the stimulated signals are converted to discriminating standard signals which meet the requires of the test instrument. The different test points are connected to revenant signals modulator according to the signal kinds by signals connecting circuit board, each connecting board is fixed on the instructions receiver trestle.

The adapter can be communicated real timely with the main computer through RS-232 bus, the signals' generation and modulation can be completed by controlling each signal modulation subassembly according to the orders which sent by computer. In order to complete signals modulation better, isolating signals, flitting, the adapter is separated from the interface circuit board. The radar signals receiver's signals ports are connected to the interface circuit board through the standard cable; they are used to complete the signal connection with adapter. The cable tie-in signals are connected to the signals modulation adapter ports though the shield cables based on influencing intrinsic signal transform channels [12].

\section{Software Design}

The program design is the core of the system software; it is composed of operation system flat roof, application program exploitation flat roof, and test application program. The frame of it is shown in Fig.3.

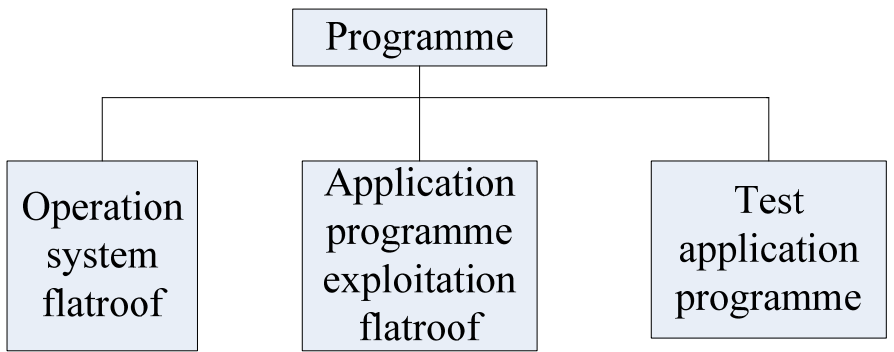

Fig.3. the program frame

Operation System Flat roof. Operation system flat roof is the basically falt roof of the application program exploitation flat roof and the test application program, its capability will influence directly the test system's functions and capability. The Windows NT is adopted by software system, the Windows NT flat roof has many advantages such as simply legible, high reliability, good security, high compatibility and so on, so it is the preferred operation flat roof, it is used to manage the whole system's hardware and software resources.

Application Program Exploitation Flat roof. Lab Windows/CVI 5.0 of NT Company is chosen as the application program exploitation flat roof. Lab Windows/CVI 5.0 is a dummy instrument exploitation flat roof face to computer test control region, it is exploited by the NT company, it's laid the base function of GPIB, VXI, serial interface, and insert mode DAQ board, it can provide 
deft and convenient interface making, program, the program's workload is predigested. It has advanced analysis base, it can complete signals proposal, statistic, curve face analog systemization and complicated analysis work. In order to make up Lab Windows/CVI software's some localization, Windows SDK, Photoshop and Visual C++ soft wares are adopted to complete other assistant work.

Test Application Program. According to the instrument's requires, modulization design method is adopted in test application program, modules include instrument test self module, performance test module, instrument drive module, data management module, faults diagnosis module.

The system test self module is used to complete the whole test system's test self function, the system faults diagnosis and isolation can be completed by depending to the system interior itself circuit and program. All the hardware circuits beside the computer, can be carried test self under the control of such module, the results can be shown. If the faults happen, the fault parts can be shown, users can deal with faults through revenant methods.

The capability test module is divided according to the radio's circuit functions, it is composed of receiver module, control box module, basic electric parameters module. Each module includes the radio test items. After the item is chosen by users, the program will carry the revenant function, the chosen item is tested. The program can be carried out by two methods, one is auto test, and the other is option test. Auto test method can complete auto test according to the items which is set according to capability test maintenance techniques. Option test module is used to test the single item, at the same time, such module is used to estimate and show the test results.

The data management module is an independent module, when the test device's capability test is completed; the test results are managed by the data management module as the form of database files. Such module includes results query and browse, results deleting and refiguring, results printing and so on. Such module is provided to users as graphics mode interface, it includes results query window, results browse window, results refigure window, results print window and so on.

The instrument drive module includes CMS-50 integrated test instrument drive program, three-purpose meter, and Windows NT bottom interrupt drive program and so on. Generally, drive program is at the software system's bottom, it can be loaded and transferred by capability test operation module.

\section{Key Techniques}

The Real-time Test Technique. During test, the auto-pilot system is used to test every kind of the power parameter, the voltage current, and the rudder deflexion angle and ready time.

When the test system is used to test every parameter, the test parameter location point need be choose. The test point number and sensitivity is associated with the detection ratio and the isolation ratio of fault diagnosis.

The test devices are mainly the three axes revolving platform and the rudder deflexion angle protractor. The former is used to simulate the athletic gestures of pitching, yaw and roll when the missile flies in the sky, however the rudder deflexion angle protractor is mainly used to test the deflexion angle of four rudders, the test cycle may controlled by the software[13].

Anti-jamming technique based on combining between software and hardware. The missile test environment is complicated, and most of the devices are high frequency signals, the electromagnetism environment is very bad, so the key questions of design and manufacture are the test system's stability and reliability. During the manufacture process, some systems which work good in the lab are found that they can't work or work bad when they are transformed to the test locale. So when the system is designed and machined, such method should be used as replacing LCD, adopting photo-electricity isolation, low-pass filter to improve the system's anti-jamming capability, at the same time, the redundancy technique is used in the software design, in this way, the thing which the program can't be run because of the jam will be prevented farthest, the stability and security of the program is improved.

The hardware anti-jamming technique. Aim at the signal process channels characteristic, next methods are adopted in the hardware design. 
Photo-electricity coupling isolation. Aim at the frequency signal transmission of the tested missile auto-pilot system, the photo-electricity coupling isolation method is used. In fact, the signals which bring strong jam to the frequency signal are aiguilles signals, such kind of signal can't make the light-coupling implement irradiancy to produce effective signals, so such kind of signal can be isolated effectively by using he photo-electricity coupling isolation technique.

Isolation amplifier. The output signal of the missile test signal is small; however the ordinary light-coupling implement has higher linearity, if it is used to transfer such above signal directly, the precision is low, so the isolation amplifier is used to isolate. The stability and linearity of the isolation amplifier is very good, at the same time, it has high restrain ratio, alterable amplification plus, so it is fit to be used to isolate and reduce noise[14].

High pass filter. According to frequency range analysis of the system work signal, the noise mainly comes from the low frequency jam. So the high pass filter can isolate the noise effectively.

The software anti-jamming technique. Software time-lapse technique. The analog signals which collected by A/D will be switched through the multiplex switches, at the beginning, the analog signals dithering will happen, after a few minutes, the dithering will disappear. In order to eliminate the dithering, the software time-lapse method is adopted, that is the time-lapse control sentences are added in the program code when the signals are sent and the A/D is started, in this way, and sampling precision is improved effectively[15].

Software filter technique. In order to improve the capability of anti-jamming and anti-noise, to ensure the test precision, the metrical value is processed by the digital filter, which is to obtain the average value after 256 times measurement. Such above method is easily to complete. After the system is processed by the software and hardware, the error of the system is only \pm 1 LSB.

\section{Conclusion}

The target of radar signals receiver auto test system is portable and expandable, the hardware and software are designed detailed. The practice results illustrate that such system's capabilities are reliable, the operation interface is friendly, the use maintenance is easily, the missile's test parameters are record and analyzed exactly, the missile parameters' test precision and test work efficiency are improved, the missile radar signals receiver test efficiency is enhanced obviously, it has remarkable military and economical benefits.

\section{References}

[1] Yong Yu, Chi-Leung Hu, Tsan-Ming Choi. An Empirical Study of Intelligent Expert Systems on Forecasting of Fashion Color Trend [J]. Expert System with Applications, 2012(39) 4383-4389.

[2] Hossien Riahi-Madvar, Seyed Ali Ayyoubzadeh, Mina Gholizadeh Atani. Developing an expert system for predicting alluvial channel geometry using ANN. Expert System with Application [J], 2011(38) 215-222.

[3] Xia Yuhang, Tenghuan, Fengchao. Study on Electronic Attemperation Relation Based on Modified BP Network. Electro technical Application [J], 2012 31(17) 81-85,

[4] Ren Weijian, Wang Chongyun, Kang chaohai. Fault Diagnosis Technology Based on Neural Network and Expert System. Petrochemical Electric [J], 2013(32) 66-71,

[5] Wanghui, Li Xiaoquan, Zeng Jiangdong. Fault Diagnosis of the Power Vehicle Based on Rough Set Theory. Movable Power Station \& Vehicle [J]. 2011(3) 12-15,.

[6] Zhang Zhenshan, Gao Ziheng, Fan Jianling. Design and Application of Neural Network Expert System Based on VB. Computer Test and Control, 2008 16(8) 1099-1100,.

[7] Zhang Defeng. MATLAB Neural Network Application Design. Beijing: Mechanic Industry Press [M], 2009. 
[8] Fengding. The Neural Network Expert System. Beijing: Science Press [M], 2006.

[9] Ken-ichi Funahashi. On the Approximate Realization of Continuous Mappings by Neural Networks. Neural Network [J], 1989(2) 183-192.

[10]Veera Babu K, Ganesh Narayanan R, Saracana Kumar G. An Expert System Based on Artificial Neural Network for Predicting the Tensile Behavior of Tailor Welded Blanks. Expert System with Applications [J], 2009(36) 10683-10695.

[11] Li Bicheng, Luo Jianshu. Wavelet Analysis and Application. Beijing: Electrical Industry Press [M], 2003.

[12] Gu Shuiqing. Electric Power System Relay Safeguard. Beijing: China Electric Power Press [M], 2005.

[13] Cybenko G. Continuous Valued Neural Networks with Two Hidden Layers are Sufficient. Mathematics of Control, Signals, and Systems [J], 1989(2) 330-341.

[14] Xiao Mingqing, Wang Xueqi. Airborne Missile Test Theory. Beijing: National Defence Industry Press [M], 2011.

[15] Zhang Jie, Gao Xianjun, Yao Jinbo. Fault Diagnosis Technology Based on Neural Network and Expert System. Journal of Jilin University [J], 2009 27(3) 321-324. 